\title{
SCHUBERT CALCULUS IN COMPLEX COBORDISM
}

\author{
PAUL BRESSLER AND SAM EVENS
}

\begin{abstract}
We study the structure of the complex cobordism ring of the flag variety of a compact connected Lie group. An explicit procedure for determining products of basis elements is obtained, generalizing the work of Bernstein$\mathrm{Gel}^{\prime}$ fand-Gel'fand on ordinary cohomology and of Kostant-Kumar on $K$ theory. Bott-Samelson resolutions are used to replace the classical basis of Schubert cells.
\end{abstract}

\section{INTRODUCTION}

Let $G$ be a connected compact Lie group with maximal torus $T$ and let $X=G / T$ be its generalized flag variety. The study of the geometry and topology of $X$ is crucial for a number of problems related to the representation theory and topology of $G$. An important aspect of this study is the intersection theory of algebraic cycles on $X$, classically known as the Schubert calculus. In the case where $G=U(n)$, this problem generalizes the nineteenth century work of Schubert on intersections of algebraic cycles in the Grassmannian.

A 1973 paper of Bernstein, Gel' fand, and Gel'fand [BGG] gave a procedure for determining the intersection pairings in terms of the rational cohomology of $X$ (see also [D]). This work extended results of Borel, Bott and Samelson, and Kostant and has recently been extended to Kač-Moody groups by Kostant and Kumar (see also [A] and [K]). Kostant-Kumar [KK] studied an analogous problem for the complex $K$-theory of the flag variety. Much of this work focused on the algebro-geometric properties of the flag variety.

In this paper we take a more topological point of view. As a consequence we are able to extend the classical results to all complex-oriented multiplicative cohomology theories. Examples of such theories are ordinary cohomology, $K$ theory, elliptic cohomology, and complex cobordism. These additional theories contain more subtle topological information, which is interpreted in terms of the coefficients of their formal group laws. In addition, we are able to unify the study of ordinary cohomology and $K$-theory, which previously had been studied by parallel but distinct methods.

The problem of the Schubert calculus may be described as follows. The flag variety is a smooth projective variety, endowed with the structure of a CWcomplex by the Bruhat cellular decomposition. The cells $X_{w}$ (called Schubert

Received by the editors March 20,1990. Presented at the AMS special session on classical and quantum groups, April 8, 1990, University Park, Pennsylvania.

1980 Mathematics Subject Classification (1985 Revision). Primary 57T15, 55N22.

The second author was supported by Sloan Foundation dissertation year fellowship. 
cells) are indexed by elements $w$ of the Weyl group $W$. The dimension of the cell $X_{w}$ is equal to twice the length of the corresponding word. The Schubert cells form a free basis for the cohomology of $G / T$ and the classical Schubert calculus determines the multiplicative structure of $H^{*}(G / T ; Z)$ in terms of this basis. In the analogous problem for $K$-theory cells are represented by their structure sheaves.

In general the cells $X_{w}$ do not determine classes in $h^{*}(G / T)$ for an arbitrary generalized cohomology theory. However, for a multiplicative cohomology theory $h^{*}$ with complex orientation we construct a family of elements in $h^{*}(G / T)$ which

1. generalize the classes determined by $X_{w}$ (in ordinary cohomology and $K$-theory);

2. generate $h^{*}(G / T)$ as a module over the ring of coefficients $h^{*}$;

3. arise from classical geometric objects.

These elements descend to $h^{*}(G / T)$ from $M U^{*}(G / T)$ under the complex orientation $M U \rightarrow h$. In $M U^{*}(G / T)$ they are represented by the Bott-Samelson resolutions of singularities.

An algebra generated by operators which are in one-to-one correspondence with the simple reflections in the Weyl group acts on $h^{*}(G / T)$. In fact any resolution class arises by applying a product of these operators to the resolution of the zero dimensional Schubert variety. Using a geometric description of the operators we define analogous operators on $h^{*}(G / T)$. The formula of BrumfielMadsen for transfer in bundles of homogeneous spaces yields an expression for the operators in terms of the Euler classes of line bundles on $G / T$. The formulas which we obtain generalize those known in cohomology and $K$-theory.

The operators enable us to express the resolution classes in terms of the characteristic classes. We also describe a recursive procedure for calculation of products of resolution classes with the Euler classes of line bundles which leads to a procedure for calculation of products of resolution classes. The algorithm yields Schubert calculus for any complex oriented cohomology theory but differs from the known algorithm for complex $K$-theory.

The classical operators from ordinary cohomology and $K$-theory satisfy the so-called braid relations. In a previous paper, we showed that these two theories were essentially the only theories in which braid relations were satisfied. As a consequence of this fact, two different resolutions for the same Schubert variety may define different classes in $h^{*}(G / T)$, if $h$ is not ordinary cohomology or $K$ theory. However, one can still determine the product of two resolution classes in terms of a basis. The difference between two resolutions may be regarded as more delicate information about the resolutions than appears in cohomology or $K$-theory.

This paper is organized as follows. After recalling some facts about multiplicative cohomology theories with complex orientation we give a short account of Quillen's geometric interpretation of complex cobordism. In the following sections we use this description of $M U$ to define a family of classes in $M U^{*}(G / T)$ and give a procedure for calculating cup and cap products. The resulting formulas describe Schubert calculus for any multiplicative cohomology theory with complex orientation. 


\section{COMPLEX ORIENTED COHOMOLOGY THEORIES}

In this section we recall the basic properties of multiplicative generalized cohomology theories with complex orientation.

A multiplicative cohomology theory $h$ is a functor from topological spaces to graded rings satisfying the Eilenberg-Steenrod axioms except for the dimension axiom and some additional axioms for the product [Dy]. A multiplicative cohomology theory $h$ is complex oriented if complex vector bundles are oriented for $h$. In a complex oriented cohomology theory the Euler class and the Chern classes of complex vector bundles are defined and satisfy the usual properties. Examples of complex oriented cohomology theories are ordinary cohomology, complex $K$-theory, elliptic cohomology, and complex cobordism. Complex cobordism is the universal complex oriented theory, so for any such theory there is natural transformation $M U \rightarrow h$.

A complex orientation of a proper map of (smooth) manifolds

$$
f: M \rightarrow N
$$

is a factorization

$$
M \stackrel{i}{\hookrightarrow} E \stackrel{\pi}{\rightarrow} N
$$

where $E$ is a complex vector bundle and $i$ as an embedding with a stably complex normal bundle. A compact manifold is said to be complex oriented if and only if the map to a point is complex oriented (equivalently the tangent bundle is stably complex).

Let $h$ be a complex oriented theory. A proper complex oriented map

$$
f: M \rightarrow N
$$

induces the Gysin homomorphism

$$
f_{*}: h^{*}(M) \rightarrow h^{*-k}(N)
$$

of degree $k=\operatorname{dim} M-\operatorname{dim} N$.

Given proper choices of complex orientations the Gysin homomorphism satisfies the following properties:

1. The projection formula holds (i.e. with notations as above $f_{*}$ is a map of $h^{*}(N)$ modules).

2. Naturality (i.e. $\left.(f \circ g)_{*}=f_{*} \circ g_{*}\right)$.

3. Base change. Consider a Cartesian square

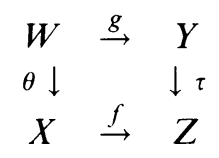

Then $f^{*} \circ \tau_{*}=\theta_{*} \circ g^{*}$.

To a cohomology theory $h$ with complex orientation corresponds a formal group law defined over the coefficient ring $h^{*}$, i.e. a power series $\mathscr{F}$ over $h^{*}$. The group law expresses the Euler class of the tensor product of two complex line bundles in terms of the Euler classes of each, so

$$
\chi\left(L_{1} \otimes L_{2}\right)=\mathscr{F}\left(\chi\left(L_{1}\right), \chi\left(L_{2}\right)\right) .
$$

For example the group law of ordinary cohomology is additive, i.e.

$$
\mathscr{F}(X, Y)=X+Y,
$$


and the group law of complex $K$-theory is multiplicative, i.e.

$$
\mathscr{F}(X, Y)=X+Y+u X Y,
$$

where $u$ is a unit. As Quillen showed in [Q] the group law associated with complex cobordism is the universal group law.

\section{GEOMETRIC INTERPRETATION OF COMPLEX COBORDISM}

In this section we recall a geometric definition of complex cobordism and of the corresponding homology theory of complex bordism and describe the associated notions (e.g. the induced maps, products, duality) in terms of these definitions.

We define the (co)bordism groups for a smooth manifold (not necessarily compact). All maps will be assumed to be smooth.

Complex bordism theory $M U$ was originally defined by geometric means as bordism classes of maps of stably complex manifolds. More precisely for a space $X$ the underlying set of the group $M U_{q}(X)$ is the set of equivalence classes of maps

$$
M \stackrel{f}{\rightarrow} X \text {. }
$$

Here $M$ is a closed, stably almost complex manifold. This means that $M$ is a compact smooth manifold without boundary of dimension $q$ with $\mathrm{T} M$ stably complex. Two such maps $(M, f)$ and $(N, g)$ are equivalent (bordant) if and only if their disjoint union extends to a map

$$
W \rightarrow X
$$

of a compact stably almost complex manifold $W$ of dimension $q+1$, whose boundary is the union of $M$ and $N$. Moreover, the stably almost complex structures on $M$ and $N$ induced by the embedding into $W$ and the original ones are required to be equivalent. The Abelian group structure on $M U_{*}(X)$ is given by the operation of disjoint union. By a theorem of Rene Thom the resulting groups coincide with those obtained by a homotopy-theoretic construction [S].

The dual cohomology theory $M U$ called complex cobordism was given a geometric description by D. Quillen [Q]. We present an outline of his construction below.

For a manifold $X$ of dimension $n$ an element in $M U^{n-q}(X)$ is represented by a differentiable, proper map

$$
M \rightarrow X
$$

of a (not necessarily compact) manifold $M$ of dimension $q$ together with an equivalence class of complex orientations. Two such maps are equivalent if and only if they are bordant as maps with complex orientations.

A differentiable map of manifolds

$$
X \stackrel{g}{\rightarrow} Y
$$

induces a map

$$
g_{*}: M U_{*}(X) \rightarrow M U_{*}(Y)
$$

in bordism by composing: $g_{*}[(M, f)]=[(M, g \circ f)]$. It also induces a map

$$
g^{*}: M U^{*}(Y) \rightarrow M U^{*}(X)
$$


in cobordism defined as follows. Let $(M, f)$ represent an element in $M U^{*}(Y)$. The map $f$ can be chosen to be transverse to $g$. Then $g^{*}[(M, f)]$ is represented by the left vertical arrow in the diagram

$$
\begin{array}{ccc}
M \times_{Y} X & \rightarrow & M \\
\downarrow & & \downarrow f \\
X & \stackrel{g}{\rightarrow} & Y
\end{array}
$$

Complex cobordism is a multiplicative cohomology theory. The external product

$$
M U^{*}(X) \otimes M U^{*}(Y) \rightarrow M U^{*}(X \times Y)
$$

is defined by taking the Cartesian product of maps. For a space $X, M U^{*}(X)$ is a (graded) commutative ring with unit under cup product defined by

$$
[(M, f)][(N, g)]=\Delta^{*}[(M \times N, f \times g)],
$$

where $\Delta$ is the diagonal embedding of $X$ into $X \times Y$. Thus the cup product is represented by the "geometric intersection of cycles." The unit element is represented by the identity map. Similarly there is a cap product pairing

$$
M U^{*}(X) \otimes M U_{*}(X) \rightarrow M U_{*}(X)
$$

given by the fiber product of maps.

A compact stably almost complex manifold $X$ has a fundamental class in $M U_{*}(X)$ represented by the identity map. Poincare duality can be described as follows. Let $(M, f)$ represent an element in $M U^{*}(X)$. The complex orientation of $f$ and the stably almost complex structure on $X$ induce a stably almost complex structure on $M$. With this structure $(M, f)$ represents an element in $M U_{*}(X)$ of complementary (with respect to the dimension of $X$ ) degree which is the Poincaré dual.

For a proper complex oriented map

$$
X \stackrel{f}{\rightarrow} Y
$$

the Gysin map

$$
f_{*}: M U^{*}(X) \rightarrow M U^{\star-(\operatorname{dim} X-\operatorname{dim} Y)}(Y)
$$

is defined by composing with $f$. In presence of Poincare duality this is the adjoint to the induced map in bordism.

Stably complex vector bundles are oriented in complex cobordism. For a vector bundle $E \rightarrow X$ with the zero section $\zeta$ the Thom class $U_{E}$ in $M U^{*}\left(X^{E}\right)$ is defined by $U_{E}=\zeta_{*}(1)$, where $X^{E}$ denotes the Thom space of $E$ and $\zeta$ denotes the zero section. The Euler class $\chi(E)$ in $M U^{*}(X)$ is defined by $\chi(E)=\zeta^{*} \zeta_{*}(1)$. Thus the Euler class is represented by the inclusion of the submanifold of zeros of a generic section.

\section{GEOMETRY AND TOPOLOGY OF THE FLAG VARIETY}

In what follows $G$ is a compact simply-connected Lie group. We fix a maximal torus $T \subset G$. The complexified Lie algebras of $G$ and $T$ will be denoted by $\mathbf{g}$ and $\mathbf{t}$ respectively. Let $\mathscr{R}$ denote the set of roots of $G$ and choose a system of positive roots $\mathscr{R}^{+}$with simple roots $\Delta=\left\{\alpha_{1}, \ldots, \alpha_{l}\right\}$. The compact group $G$ can be embedded in a complex group $G_{\mathbf{C}}$ with Lie algebra $\mathbf{g}$. In $G_{\mathbf{C}}$ we choose the Borel subgroup $B$ containing $T$ and having $\mathscr{R}^{+}$for the roots. 
The isomorphism

$$
G / T \rightarrow G_{\mathbf{C}} / B
$$

induces a complex structure on $G / T$. The tangent bundle of the flag manifold decomposes into a sum of line bundles

$$
\mathbf{T} G / T=\bigoplus_{\alpha \in \mathscr{R}^{+}} L(-\alpha) .
$$

Here and in what follows the line bundle $L(\lambda)$ is defined by

$$
L(\lambda)=G \times{ }_{T} \mathbf{C}_{\lambda}
$$

for an integral weight $\lambda$ of $\mathbf{t}$ (cf. [BH]).

Let $W$ denote the Weyl group of $G$. The flag variety decomposes into a union of $B$-orbits (under the left $B$-action):

$$
G_{\mathbf{C}} / B=\bigcup_{w \in W} X_{w}
$$

where $X_{w}=B w B$ and $\operatorname{dim} X_{w}=2 l(w) \quad(l(w)$ denotes the length of the word $w$ ) [BGG]. This decomposition gives $G / T$ a structure of a $\mathrm{CW}$ complex. The closure $\bar{X}_{w}$ of the cell $X_{w}$ is an algebraic subvariety of $G / T$ (often singular) called a Schubert variety. Its boundary is a union of cells corresponding to all the words which are smaller than $w$ in Bruhat ordering [BGG, §2]. Consequently the integral cohomology of the flag variety is free with basis $\bar{X}_{w}, w \in W$ and is concentrated in even degrees.

Since $M U^{*}$ is also even graded the Atiyah-Hirzebruch spectral sequence

$$
H^{*}\left(G / T ; M U^{*}\right) \Rightarrow M U^{*}(G / T)
$$

collapses at the $E_{2}$ term and there is an isomorphism of abelian groups

$$
M U^{*}(G / T) \cong H^{*}(G / T) \otimes M U^{*} .
$$

It follows from the universal property of $M U$ that we can restrict our attention to the complex cobordism theory. Indeed, by naturality of the AtiyahHirzebruch spectral sequence, the above spectral sequence collapses at the $E_{2}$ term for any complex oriented theory $h$. The ring structure of $h^{*}(G / T)$ is obtained by specialization using the Thom class.

The Schubert cycles $\bar{X}_{w}$ which generate the associated graded group $H^{*}(G / T) \otimes M U^{*}$ of $M U^{*}(G / T)$ do not have canonical liftings to cobordism classes. However, we can use the resolutions of singularities of Schubert varieties to represent liftings. We will use cobordism classes given by Bott-Samelson resolutions of singularities of Schubert varieties (also known as the canonical resolutions). They appeared in the context of Schubert calculus in the work of Bott-Samelson [BS], Demazure [D], and Arabia [A].

The Weyl group $W$ of $G$ is generated by reflections $s_{1}, \ldots, s_{l}$ which are in one to one correspondence with the simple roots. We shall also consider subgroups $H_{1}, \ldots, H_{l}$ of $G$ of minimal rank. $H_{k}$ can be described as the subgroup containing $T$ with roots $\alpha_{k}$ and $-\alpha_{k}$. The complexified Lie algebra of $H_{k}$ is $\mathbf{g}_{\alpha_{k}} \oplus \mathbf{t} \oplus \mathbf{g}_{-\alpha_{k}}$, where $\mathbf{g}_{\alpha}$ denotes the $\alpha_{k}$ root space. There is an embedding

$$
S U(2) \rightarrow H_{k}
$$


such that the intersection of the image of the maximal torus of $S U(2)$ with $T$ is the one parameter subgroup of $T, t \mapsto \exp \left(t \lambda_{k}^{*}\right), \lambda_{k}^{*}$ denoting the element of $\mathbf{t}$ dual to the fundamental weight $\lambda_{k}$. We shall identify the flag manifold of $S U(2)$ with $\mathbf{C} \mathbf{P}^{1}$, thinking of the latter as the projectivization of the standard representation of $S U(2)$. The inclusion of $S U(2)$ into $H_{k}$ induces isomorphism of the respective flag manifolds.

We give a brief account of the construction and the properties of the BottSamelson resolutions below. A detailed study is presented in the paper by Bott and Samelson in Chapter 3.

Let $I=\left(i_{1}, \ldots, i_{n}\right)$ be a multi-index with $1 \leq i_{j} \leq l$. The space $R_{I}$ of the resolution is given by

$$
R_{I}=H_{i_{1}} \times_{T} H_{i_{2}} \times_{T} \cdots \times_{T} H_{i_{n}} / T
$$

where $T$ acts by multiplication on the right on $H_{i_{k}}$ and by multiplication on the left (by the inverse) on $H_{i_{k+1}}$. There is a map

$$
R_{I} \stackrel{\tau_{I}}{\rightarrow} G / T
$$

induced by group multiplication:

$$
\left(h_{1}, \ldots, h_{n}\right) \mapsto h_{1} \ldots h_{n} \cdot T
$$

Suppose the multi-index $I$ indexes a decomposition of a word $w \in W$ into a product of simple reflections

$$
w=s_{i_{1}} \cdot s_{i_{2}} \cdots s_{i_{n}}
$$

with $l(w)=n$ (such a decomposition is called reduced). Then $\left(R_{I}, r_{I}\right)$ is a resolution of singularities of $\bar{X}_{w}$, i.e., $R_{I}$ is smooth and the map $r_{I}$, is proper and birational [D].

Let $I$ be as before and let $J=\left(i_{1}, \ldots, i_{n-1}\right)$. Then $R_{I}$ is fibered over $R_{J}$ by $\left(h_{1}, \ldots, h_{n}\right) \mapsto\left(h_{1}, \ldots, h_{n-1}\right)$ with fiber $H_{i_{n}} / T$ isomorphic to $\mathbf{C P}^{1}$. We now give an alternative description of $R_{I}$. Consider the space $H_{i_{1}} \times_{T}$ $\cdots \times_{T} H_{i_{n-1}} \times_{T} H_{i_{n}}$. This is a principal $H_{i_{n}}$ bundle over $R_{J}$. The group $H_{i_{n}}$ has an irreducible complex two dimensional representation with highest weight $\lambda_{i_{n}}$, which, when restricted to $S U(2)$ (cf. the description of $H_{i_{n}}$ ), is just the standard representation. The associated vector bundle $V\left(J, i_{n}\right)$ over $R_{J}$ splits into the sum of line bundles:

$$
V\left(J, i_{n}\right)=L\left(\lambda_{i_{n}}\right) \oplus L\left(\lambda_{i_{n}}-\alpha_{i_{n}}\right),
$$

where $L(\lambda)$ denotes the line bundle on $R_{J}$ associated to the character $e^{\lambda}$ of the right-most torus in the definition of $R_{J}$ (and is equal to $r_{J}^{*} L(\lambda)$ ). Then $R_{I}$ is isomorphic to the projectivization of $V\left(J, i_{n}\right)$.

This makes $h^{*}\left(R_{I}\right)$ easily computable for any complex oriented theory $h$ by standard techniques. For example $h^{*}\left(R_{I}\right)$ is generated (as an algebra over the ring of coefficients) by the degree two part. The latter fact plays an important role in what follows.

The resolution $R_{I}$ is a complex manifold and the map $r_{I}$ is a holomorphic map, and, therefore, naturally complex oriented. 
Definition. $Z_{I}$ is the complex cobordism class represented by the pair $\left(R_{I}, r_{I}\right)$. By $Z_{e}$ we shall denote the cobordism class represented by the inclusion of the zero dimensional cell which corresponds to the empty multi-index.

The resolution class corresponding to a reduced decomposition of $w \in W$ descends to the class of the Schubert variety $\bar{X}_{w}$ in ordinary cohomology and complex $K$-theory. In ordinary cohomology this is an immediate consequence of the birationality of the map $r_{I}$. In $K$-theory this is a subtle fact which follows from the normality of $\bar{X}_{w}$ and vanishing of certain derived functors (cf. e.g. $[\mathrm{Ku}]$ ). In ordinary cohomology, if the multi-index $I$ indexes a nonreduced product of reflections the corresponding resolution class descends to zero. The cobordism classes corresponding to different choices of reduced decompositions are different and, in fact, usually specialize to different classes under the Thom class map. This is related to the results in [BE2] (see the remark at the end of the paper). The facts that the ordinary cohomology is free on the basis of the Schubert classes, and that the Atiyah-Hirzebruch spectral sequence degenerates at $E_{2}$, imply

Proposition 1. The collection of cobordism classes $Z_{I}$ generates $M U^{*}(G / T)$ as a module over the coefficient ring. In fact it is freely generated by any subcollection, containing exactly one class $Z_{I}$ per element $w \in W$, so that I indexes a reduced decomposition of $w$.

The flag variety naturally maps to the classifying space of the maximal torus. This map can be described as the inclusion of the fiber of the bundle

$$
B T \rightarrow B G .
$$

Alternatively, it is the classifying map of the $T$-principal bundle

$$
G \rightarrow G / T \text {. }
$$

The induced homomorphism in cohomology is called the "characteristic homomorphism"

$$
\chi: H^{*}(B T) \rightarrow H^{*}(G / T) .
$$

The cohomology ring of $B T$ with complex coefficients can be naturally identified with the completion of the ring of complex valued polynomial functions on the complexified Lie algebra of $T$, where the linear functions are assigned degree two. The integral linear functions are weights $\lambda$ of $\mathbf{t}$, which exponentiate to characters $e^{\lambda}$ on $T$. The characteristic homomorphism maps a weight $\lambda$ to the Euler class of the line bundle $L(\lambda)$ on $G / T$ associated to the character $e^{\lambda}$.

The characteristic homomorphism is surjective in rational cohomology. Consequently it is surjective in any complex oriented theory $h$ whose coefficients form an algebra over the rational numbers. In that case there is a short exact sequence

$$
0 \rightarrow\left(h^{*}(B T)_{+}^{W}\right) \rightarrow h^{*}(B T) \rightarrow h^{*}(G / T) \rightarrow 0
$$

which identifies the kernel of the characteristic homomorphism with the ideal in $h^{*}(B T)$ generated by the Weyl group invariants of positive degree.

\section{Operators ON $M U^{*}(G / T)$}

In this section we define operators $A_{i}$ which generate an algebra acting on $M U^{*}(G / T)$. This action makes $M U^{*}(G / T)$ into a cyclic module generated 
by the class $Z_{e}$ represented by the inclusion of the zero dimensional cell. The operators allow us to express the resolution classes in terms of the image of the characteristic homomorphism. They will be used in conjunction with the cap product formula to describe the multiplicative structure of $M U^{*}(G / T)$.

For each $i=1, \ldots, l$ there is a holomorphic fiber bundle

$$
\pi_{i}: G / T \rightarrow G / H_{i}
$$

with fiber isomorphic to $\mathbf{C P}^{1}$. Using these we define a family of operators $A_{i}$ $\left(M U^{*}\right.$-module endomorphisms) on $M U^{*}(G / T)$ by

$$
A_{i}=\pi_{i}^{*} \circ \pi_{i *}: M U^{*}(G / T) \rightarrow M U^{*-2}(G / T)
$$

These operators appear in the work of Bernstein-Gelfand-Gelfand, KostantKumar, and Demazure and play an essential role in Schubert calculus, as a consequence of the following proposition.

Proposition 2. Let $I=\left(i_{1}, \ldots, i_{n}\right)$ and $J=\left(i_{1}, \ldots, i_{n+1}\right)$. Then

1. $A_{i_{n+1}} Z_{I}=Z_{J}$

2. $A_{i}^{2}=A_{i}(1) A_{i}$.

Proof. 1. By definition $\pi_{i_{n+1}} Z_{I}$ is represented by the map $\left(R_{I}, \pi_{i_{n+1}} \circ r_{I}\right)$. Since $\pi_{i_{n+1}}$ is a submersion it is transverse to $\pi_{i_{n+1}} \circ r_{I}$. Therefore $A_{i_{n+1}} Z_{I}$ is represented by the left vertical arrow in the diagram

$$
\begin{array}{ccc}
H_{i_{1}} \times{ }_{T} \cdots \times \times_{T} H_{i_{n}} / T \times \times_{G / H_{i_{n+1}}} G / T & \rightarrow & R_{I} \\
\downarrow & & \downarrow \pi_{i_{n+1}} \\
G / T & \stackrel{\pi_{i_{n+1}}}{\longrightarrow} & G / H_{i_{n+1}}
\end{array}
$$

There is an isomorphism

$$
\begin{aligned}
H_{i_{1}} \times T & \cdots \times \times_{T} H_{i_{n+1}} / T \stackrel{\sim}{\rightarrow} H_{i_{1}} \times_{T} \cdots \times_{T} H_{i_{n}} / T \times_{G / H_{i_{n+1}}} G / T, \\
\left(h_{i_{1}}, \ldots, h_{i_{n+1}}\right) & \mapsto\left(h_{i_{1}}, \ldots, h_{i_{1}} \cdot h_{i_{2}} \cdots h_{i_{n+1}}\right) .
\end{aligned}
$$

2. This follows directly from the projection formula.

Corollary 1. $Z_{I}=A_{i_{1}} \circ \cdots \circ A_{i_{n}} Z_{e}$, where $Z_{e}$ denotes the class represented by the inclusion of the zero dimensional Schubert variety.

Corollary 2. The operators $A_{i}$ acting on ordinary cohomology satisfy $A_{i}^{2}=0$.

Proof. This follows from the observation that $A_{i}(1)$ is an element of degree -2 of the ring of coefficients.

The operators defined above allow us to relate the classes of the form $Z_{I}$ to the characteristic classes of line bundles on $G / T$ as follows. For a complex vector bundle $E$ let $\chi(E)$ denote the Euler class of $E$ (in $M U$ ). The Euler characteristic of the flag variety is equal to the order of the Weyl group. Then

$$
|W| Z_{e}=\chi(\mathbf{T} G / T)=\prod_{\alpha \in \mathscr{K}^{+}} \chi(L(-\alpha))
$$

The first equality follows from the fact that there is a vector field with the number of simple zeros equal to the Euler characteristic. Since the flag manifold is path connected all points define the same cobordism class. The second equality follows from the Whitney sum formula. 
Proposition 2 implies that all resolution classes $Z_{I}$ are generated from $Z_{e}$ by repeated application of operators $A_{i}$. That is if $I=\left(i_{1}, \ldots, i_{n}\right)$

$$
Z_{I}=A_{i_{1}} \circ \cdots \circ A_{i_{n}} Z_{e} .
$$

Using the above expression for $Z_{e}$ we obtain

$$
Z_{I}=\frac{1}{|W|} A_{i_{n}} \cdots A_{i_{1}} \prod_{\alpha \in \mathscr{R}^{+}} \chi\left(L\left(-\alpha_{j}\right)\right) .
$$

The operators $A_{i}$ have natural liftings to operators $C_{i}$ on $M U^{*}(B T)$. Let

$$
p_{i}: B T \rightarrow B H_{i}
$$

be the $\mathbf{C P}^{1}$ fiber bundle induced by the inclusion $T \rightarrow H_{i}$. Then define

$$
C_{i}=p_{i}^{*} \circ p_{i *}: M U^{j}(B T) \rightarrow M U^{j-2}(B T) .
$$

Naturality properties imply that the characteristic homomorphism intertwines the actions of $C_{i}$ and $A_{i}$.

The operators $C_{i}$ act on $M U^{*}(B T)$ by the formula in Proposiion 3 below. The formula is derived in [BE2] using homotopy theoretic considerations.

Proposition 3 [BE2].

$$
C_{i}=\left(1+s_{i}\right) \frac{1}{\chi\left(L\left(-\alpha_{i}\right)\right)} .
$$

\section{Cup PRODUCT FORMUla AND SCHUbert CALCUlus}

In the preceding section we developed a method for expressing resolution classes in terms of the image of the characteristic homomorphism. We shall presently describe a method for computing the products of resolution classes with characteristic classes of line bundles on $G / T$. Combined with the results of the previous section this yields a method for computing products of resolution classes.

Let $I=\left(i_{1}, \ldots, i_{n}\right)$ be a multi-index with $1 \leq i_{j} \leq l$. Recall that the cobordism class $Z_{I} \in M U^{*}(G / T)$ is represented by the map

$$
r_{I}: R_{I} \rightarrow G / T \text {. }
$$

Let $L(\lambda)$ be the line bundle associated to a weight $\lambda$. It has the Euler class $\chi(L(\lambda))$ in $M U^{*}(G / T)$. The cup product formula expresses $\chi(L(\lambda)) \cup Z_{I}$ in terms of other resolution classes.

\section{Lemma 1.}

$$
\chi(L(\lambda)) \cup Z_{I}=r_{I *} \chi\left(r_{I}^{*} L(\lambda)\right) .
$$

Proof. The equality is a direct consequence of the projection formula for the Gysin homomorphism. We can rewrite the right-hand side as

$$
r_{I *} \chi\left(r_{I}^{*} L(\lambda)\right)=r_{I *} r_{I}^{*} \chi(L(\lambda))=\chi(L(\lambda)) \cup r_{I *}(1)=\chi(L(\lambda)) \cup Z_{I} .
$$

Lemma 1 shows that the cup product formula comes from a formula for Euler classes in $M U^{*}\left(R_{I}\right)$. The line bundle $r_{I}^{*} L(\lambda)$ will be simply denoted by $L(\lambda)$. 
We establish some notation which will be used to perform calculations in $M U^{*}\left(R_{I}\right)$. Given a multi-index $I=\left(i_{1}, \ldots, i_{n}\right)$ we define new multi-indices $I_{<k}, I_{>k}, I^{k}$ by

$$
\begin{aligned}
& I_{<k}=\left(i_{1}, \ldots, i_{k-1}\right), \quad I_{>k}=\left(i_{k+1}, \ldots, i_{n}\right), \\
& I^{k}=\left(i_{1}, \ldots, i_{k-1}, i_{k+1}, \ldots, i_{n}\right) .
\end{aligned}
$$

There is a natural projection

$$
\pi_{<k}: R_{I} \rightarrow R_{I_{<k}}
$$

given by

$$
\left(h_{i_{1}}, \ldots, h_{i_{n}}\right) \mapsto\left(h_{i_{1}}, \ldots, h_{i_{k-1}}\right) .
$$

A subindex $J$ of $I$ of length $k$ is determined by a one-to-one order preserving map

$$
\sigma:\{1, \ldots, k\} \rightarrow\{1, \ldots, n\}
$$

by the rule $j_{m}=i_{\sigma(m)}$. For the subindex $J$ of $I$ of length $k$ there is a natural embedding

$$
i_{J, I}: R_{J} \rightarrow R_{I}
$$

defined by converting a $k$-tuple $\left(h_{j_{1}}, \ldots, h_{j_{k}}\right)$ to the $n$-tuple having $h_{j_{m}}$ in the $i_{\sigma(m)}$ th slot for $1 \leq m \leq k$ and the identity element elsewhere. Observe that $r_{J}=r_{I} \circ i_{J, I}$.

A pair $\left(R_{J}, i_{J, I}\right)$ represents an element of $M U^{*}\left(R_{I}\right)$. We shall denote it as well as the integral cohomology class it determines by $\left[R_{J}\right]$. In view of the observation above we have

Lemma 2. $r_{I *}\left[R_{J}\right]=Z_{J}$.

Thus the classes of the form $\left[R_{J}\right]$ are precisely the classes we use to obtain the expression for $\chi(L(\lambda))$.

A complex line bundle is determined up to isomorphism by its first Chern class $c_{1}(L)$ in integral cohomology. The group of line bundles on $R_{I}$ denoted $\operatorname{Pic}\left(R_{I}\right)$ is isomorphic to $H^{2}\left(R_{I} ; \mathbf{Z}\right)$, which is free and has a convenient basis of elements, whose liftings to $M U^{*}\left(R_{I}\right)$ can be chosen to be $\left[R_{I^{k}}\right]$.

The isomorphism

$$
\operatorname{Pic}\left(R_{I}\right) \rightarrow H^{2}\left(R_{I} ; \mathbf{Z}\right)
$$

is given by the first Chern class. $H^{2}\left(R_{I} ; \mathbf{Z}\right)$ is free with basis consisting of classes $\left[R_{I^{k}}\right]$ with $1 \leq k \leq n$. Therefore we can choose a basis for $\operatorname{Pic}\left(R_{I}\right)$ consisting of line bundles $L_{k}$, where $1 \leq k \leq n$, satisfying

$$
c_{1}\left(L_{k}\right)=\left[R_{I^{k}}\right] \text {. }
$$

Take $L_{k}$ to be the line bundle associated with the divisor $R_{I^{k}}$ (cf. [GH]). This means $L_{k}$ has a section which intersects the zero section transversally on $R_{I^{k}}$ so that

$$
\chi\left(L_{k}\right)=\left[R_{I^{k}}\right]
$$

in complex cobordism. This basis connects line bundles with a geometric basis for $M U^{*}\left(R_{I}\right)$. In ordinary cohomology, the Euler class of a line bundle on $R_{I}$ is a linear combination of $\left[R_{I^{k}}\right]$ but for a general complex oriented theory, it is a sum of products of $\left[R_{I^{k}}\right]$ computed using the formal group law associated to the theory. 
Proposition 4. Let $\lambda$ be a weight. Let $I=\left(i_{1}, \ldots, i_{n}\right)$ be a multi-index and let $s_{I}$ denote the corresponding product of reflections. Then the line bundle $L(\lambda)$ on $R_{I}$ decomposes as

$$
L(\lambda)=\bigotimes_{k=1}^{n} L_{k}^{-\left\langle s_{\left.l>k^{\lambda}, a_{i_{k}}\right\rangle}\right.}
$$

Proof. Let

$$
L(\lambda)=\bigotimes_{k=1}^{n} L_{k}^{\otimes a_{k}}
$$

be the decomposition of the line bundle $L(\lambda)$ in terms of the basis consisting of the line bundles $L_{k}$ described above. To compute the exponents $a_{k}$ we use ordinary cohomology.

Taking the first Chern class of both sides of the above formula we obtain

$$
c_{1}(L(\lambda))=\sum_{k=1}^{n} a_{k}\left[R_{I^{k}}\right]
$$

Poincare duality implies that the coefficients $a_{k}$ are exactly the solutions of the system of linear equations (over the integers) obtained by multiplying both sides of the above by the classes $\left[R_{i_{j}}\right]$ for $1 \leq j \leq n$ :

$$
c_{1}(L(\lambda))\left[R_{i_{j}}\right]=\sum_{k=1}^{n} a_{k}\left[R_{I^{k}}\right]\left[R_{i_{j}}\right]
$$

The intersection numbers which appear in the equations are determined in the Lemmas 3 and 4 following the proposition giving

$$
-\left\langle\lambda, \alpha_{i_{j}}\right\rangle=a_{j}+\sum_{k=j+1}^{n} a_{k}\left\langle\alpha_{i_{k}}, \alpha_{i_{j}}\right\rangle .
$$

Lemma 5 shows that the same system of equations is satisfied by $-\left\langle s_{I_{\supset k}} \lambda, \alpha_{i_{k}}\right\rangle$. Therefore the $a_{k}$ 's are given by

$$
a_{k}=-\left\langle s_{I_{>k}} \lambda, \alpha_{i_{k}}\right\rangle .
$$

Lemma 3. $c_{1}\left(\pi_{<k}^{*} r_{I_{<k}}^{*} L(\lambda)\right)\left[R_{i_{j}}\right]=-\left\langle\lambda, \alpha_{i_{j}}\right\rangle$ if $j<k$ and is zero otherwise.

Proof. Using the projection formula as in Lemma 1 it is easy to see that

$$
c_{1}\left(\pi_{<k}^{*} r_{I_{<k}}^{*} L(\lambda)\right)\left[R_{i j}\right]
$$

is equal to the degree of the line bundle $i_{\left(i_{j}\right), I}^{*} \pi_{<k}^{*} r_{I_{<k}}^{*} L(\lambda)$ on $R_{i_{j}}$. If $j \geq k$, then $r_{I_{<k}} \circ \pi_{<k} \circ i_{\left(i_{j}\right), I}$ is a constant map. Therefore, if $j \geq k$, the line bundle $i_{\left(i_{j}\right), I}^{*} \pi_{<k}^{*} r_{I_{<k}}^{*} L(\lambda)$ is trivial, hence of degree zero. If $j<k$, then

$$
r_{I_{<k}} \circ \pi_{<k} \circ i_{\left(i_{j}\right), I}=r_{i_{j}} .
$$

If $j<k$ consider the line bundle $r_{i_{j}}^{*} L(\lambda)$ on $R_{i_{j}}$. By Proposition 1 the map

$$
r_{i j}: R_{i_{j}} \rightarrow G / T
$$

can be identified with the inclusion

$$
i: H_{i_{j}} / T \hookrightarrow G / T \text {. }
$$


Under the identification of $H_{i_{j}} / T$ with $\mathbf{C P}^{1}$ (cf. discussion in $\S 4$ ) the line bundle $i^{*} L\left(\lambda_{i_{j}}\right)$ corresponds to $\mathscr{O}_{\mathbf{C P}^{1}}(-1)$ - the tautological line bundle of degree -1 . A line bundle associated to a weight $\lambda$ is a tensor power of $i^{*} L\left(\lambda_{i_{j}}\right)$ with exponent the multiplicity of $\lambda_{i_{j}}$ in $\lambda$ which is precisely $\left\langle\lambda, \alpha_{i_{j}}\right\rangle$. Consequently it has degree $-\left\langle\lambda, \alpha_{i_{j}}\right\rangle$.

Lemma 4. The following integral cohomology intersection numbers arise:

if $j>k$ then $\left[R_{I^{k}}\right]\left[R_{i_{j}}\right]=0$;

if $j=k$ then $\left[R_{I^{k}}\right]\left[R_{i_{j}}\right]=1$;

if $j<k$ then $\left[R_{I^{k}}\right]\left[R_{i_{j}}\right]=\left\langle\alpha_{i_{k}}, \alpha_{i_{j}}\right\rangle$.

Proof. In the second case we have a transverse intersection at $R_{e}$ (one point) so $\left[R_{I^{k}}\right]\left[R_{i_{j}}\right]=1$. In the remaining cases $R_{i_{j}}$ is contained in $R_{I^{k}}$. The normal bundle of $R_{I^{k}}$ in $R_{I}$ is easily seen to be $\pi_{<k}^{*} r_{I_{<k}}^{*} L\left(-\alpha_{i_{k}}\right)$. Then the intersection number can be computed by

$$
\left[R_{I^{k}}\right]\left[R_{i_{j}}\right]=c_{1}\left(\pi_{<k}^{*} r_{I_{<k}}^{*} L\left(-\alpha_{i_{k}}\right)\right)\left[R_{i_{j}}\right]
$$

in which case the answer follows from Lemma 3.

Lemma 5. Let $I=\left(i_{1}, \ldots, i_{n}\right)$ be a multi-index with $1 \leq i_{j} \leq l$, and let $s_{I}$ be the corresponding product of simple reflections. Then for any weight $\lambda$ the following identity holds:

$$
\lambda=s_{I} \lambda+\sum_{k=1}^{n}\left\langle s_{I_{\supset k}} \lambda, \alpha_{i_{k}}\right\rangle \alpha_{i_{k}} .
$$

Proof. The identity is easily verified by expanding $s_{I} \lambda$.

As an immediate corollary of the proposition we obtain the cap product formula in ordinary cohomology.

Theorem (cf. [BGG]). Let $\lambda$ be a weight and let $L(\lambda)$ denote the associated line bundle on $G / T$. Let $I=\left(i_{1}, \ldots, i_{n}\right)$ be a multi-index indexing a reduced decomposition of $w=s_{I}$ in the Weyl group (i.e. $\left.l(w)=n\right)$. Then the cap product of $c_{1}(L(\lambda))$ with the homology class of the Schubert variety $\bar{X}_{w}$ is given by the formula

$$
c_{1}(L(\lambda)) \cap \bar{X}_{w}=\sum_{k}-\left\langle\lambda, s_{I_{>k}}^{-1} \alpha_{i_{k}}\right\rangle \bar{X}_{s_{l} k}
$$

where $k$ satisfies $1 \leq k \leq n$ and $l\left(s_{I^{k}}\right)=l(w)-1$.

We shall now present a procedure for calculation of products $\chi(L(\lambda)) Z_{I}$ in complex cobordism. We have

$$
\chi(L(\lambda)) \cap Z_{I}=r_{I *} \chi\left(r_{I}^{*} L(\lambda)\right)=r_{I *}\left(\sum_{\alpha=\left(i_{1}, \ldots, i_{n}\right)} m_{\alpha}\left[R_{I^{1}}\right]^{f_{i_{1}}} \cdots\left[R_{I^{1}}\right]^{f_{i_{n}}}\right)
$$

for some coefficients $m_{\alpha} \in M U^{*}$ determined by the formal group law of $M U$. Thus we have to compute all products in the above summation. By induction it suffices to compute products of the form $\left[R_{I^{k}}\right]\left[R_{J}\right]$ where $J$ is a submultiindex of $I$. After we have computed these products we obtain a product free expression in the above to which we can apply $r_{I *}$ to obtain the final result in terms of classes $Z_{J}$. 
It remains to reduce the computation of $\left[R_{I^{k}}\right]\left[R_{J}\right]$ to a calculation of characteristic classes. Two cases arise, namely either $I^{k} \cup J=I$ or $J$ is a subindex of $I^{k}$. If $I^{k} \cup J=I$ the intersection is transverse and the homological intersection coincides with the set theoretic one, i.e.

$$
\left[R_{I^{k}}\right]\left[R_{J}\right]=\left[R_{I^{k} \cap J}\right] .
$$

Otherwise we have $J \subset I^{k}$ and

$$
R_{J} \stackrel{i_{J} . l^{k}}{\hookrightarrow} R_{I^{k}} \stackrel{i_{l^{k}}, l}{\hookrightarrow} R_{I} \stackrel{r_{l}}{\longrightarrow} G / T .
$$

Let $\nu$ denote the normal bundle of $R_{I^{k}}$ in $R_{I}$. Then

$$
\left[R_{J}\right]\left[R_{I^{k}}\right]=i_{J, I^{k} *} \chi\left(i_{J, I^{k}}^{*} \nu\right) \text {. }
$$

We next observe that

$$
i_{J, I^{k}}^{*} \nu=i_{J, I^{k}}^{*} \pi_{<k}^{*} r_{I_{<k}}^{*} L\left(-\alpha_{i_{k}}\right) .
$$

The following cases arise. If $J \subset I_{>k}$ then $R_{J}$ is contained in the fiber of $\pi_{<k}$. In that case $i_{J, I^{k}}^{*} \nu$ is trivial and the intersection is equal to zero. If $J \subset I_{<k}$ then

$$
\pi_{<k} \circ i_{J, I^{k}}=i_{J, I_{<k}}
$$

and

$$
i_{J, I^{k}}^{*} \nu=r_{J}^{*} L\left(-\alpha_{i_{k}}\right) .
$$

Finally if neither of the above is the case there is an index $m$ such that $J_{<m} \subset$ $I_{<k}$ and $J_{>m} \subset I_{>k}$. Then

$$
\pi_{<k} \circ i_{J, I^{k}}=i_{J_{<m}, I_{<k}} \circ \pi_{<m}
$$

and for the line bundle we have

$$
i_{J, I^{k}}^{*} \nu=\pi_{<m}^{*} i_{J_{<m}, I_{<k}}^{*} r_{I_{<k}}^{*} L\left(-\alpha_{i_{k}}\right)=\pi_{<m}^{*} r_{J_{<m}}^{*} L\left(-\alpha_{i_{k}}\right) .
$$

This completes the discussion of the cap product formula in complex cobordism.

We now have the means to express a resolution class $Z_{I}$ in terms of the characteristic classes (using the operators $A_{i}$ ) and to calculate the products of resolution classes with characteristic classes (using the cap product "formula" described above). These combined yield a method for calculation of products of resolution classes in terms of resolution classes. We summarize all of the preceding discussion in a theorem.

Theorem. Let $G$ be a compact connected Lie group with maximal torus $T$. Let $h$ be a multiplicative cohomology theory with complex orientation. Then there is an algorithmic procedure for computing products in $h^{*}(G / T)$ in the set of generators of the form $Z_{I}$. The procedure depends only on the root system data and the formal group law associated with the cohomology theory.

Remark. As a consequence of the above cap product formula we can give a more geometric proof of the fact that the braid relations are not satisfied for elliptic cohomology or cobordism (cf. [BE2, BE1, and G]). Indeed if $R_{I}$ and $R_{J}$ are two distinct resolutions of the same Schubert variety, then one easily sees that there are combinations $p$ of characteristic classes such that $p \cap Z_{I} \neq p \cap Z_{J}$. 


\section{REFERENCES}

[A] A. Arabia, Cohomologie T-equivariante de la variété de drapeaux d'un groupe de KačMoody, Bull. Soc. Math. France 117 (1989), 129-169.

[BGG] I. N. Bernstein, I. M. Gel'fand and S. I. Gel'fand, Schubert cells and the cohomology of spaces $G / P$, Russian Math Surveys 28 (1973), 1-26.

[BE1] P. Bressler and S. Evens, On certain Hecke rings, Proc. Nat. Acad. Sci. U.S.A. 84 (1987), 624-625.

[BE2] _ The Schubert calculus, braid relations, and generalized cohomology, Trans. Amer. Math. Soc. 317 (1990), 799-811.

[BH] A. Borel and F. Hirzebruch, Characteristic classes and homogeneous spaces. I, Amer. J. Math. 80 (1958), 458-538.

[BS] R. Bott and H. Samelson, Application of the theory of Morse to symmetric spaces, Amer. J. Math. 80 (1958), 964-1029.

[D] M. Demazure, Desingularization des variétés de Schubert, Ann. École Norm. Sup. (4) 7 (1974), 53-58.

[Dy] E. Dyer, Cohomology theories, Benjamin, New York, 1969.

[G] E. Gutkin, Representations of Hecke algebras, Trans. Amer. Math. Soc. 309 (1988), 269277.

[GH] P. Griffiths and J. Harris, Principles of algebraic geometry, Wiley, 1978.

[K] V. Kač, Constructing groups associated to infinite dimensional lie algebras, Infinite Dimensional Groups with Applications, MSRI Series, Springer-Verlag, 1985.

[KK1] B. Kostant and S. Kumar, The nil Hecke ring and cohomology of G/P for a Kac-Moody group $G$, Adv. in Math. 62 (1986), 187-237.

[KK2] _ , T-equivariant,$K$-theory of generalized flag varieties, Proc. Nat. Acad. Sci. U.S.A. 74 (1987), 4351-4354.

[Ku] S. Kumar, Demazure character formula in arbitrary Kač-Moody setting, Invent. Math. 89 (1987), 395-423.

[Q] D. G. Quillen, Elementary proofs of some results of cobordism theory using Steenrod operations, Adv. in Math. 7 (1971), 29-56.

[S] R. Stong, Notes on cobordism theory, Princeton Univ. Press, Princeton, N.J., 1968.

Department of Mathematics, Purdue University, West Lafayette, Indiana 47907

Department of Mathematics, University of Arizona, Tucson, Arizona 85721

E-mail address: evens@math.arizona.edu 